\title{
Effect of an aerosol box on tracheal intubation difficulty
}

\author{
Ryo Wakabayashi ${ }^{1}$ (1) $\cdot$ Takashi Ishida $^{1} \cdot$ Tomokatsu Yamada $^{1} \cdot$ Mikito Kawamata $^{1}$
}

Received: 9 June 2020 / Accepted: 24 July 2020 / Published online: 29 July 2020

(c) Japanese Society of Anesthesiologists 2020

\begin{abstract}
The aim of this study was to determine the effect of an aerosol box on tracheal intubation difficulty. Eighteen experienced anesthetists intubated the trachea of a manikin with a normal airway 6 times using a direct laryngoscope, a McGRATH ${ }^{\mathrm{TM}}$ MAC videolaryngoscope, or an airway scope AWS-S200NK videolaryngoscope with or without an aerosol box. Although the aerosol box prolonged the time to successful intubation and decreased the percentage of glottic opening (POGO) score when using a direct laryngoscope, the statistically significant differences were clinically irrelevant. When a McGRATH ${ }^{\mathrm{TM}}$ MAC and an AWS-S200NK were used, the times to successful intubation and POGO scores were comparable with and without the aerosol box. When using any of the laryngoscopes, there were no statistically significant differences in the Cormack-Lehane grade and peak force to maxillary incisors with and without the aerosol box. In summary, the effect of an aerosol box on tracheal intubation difficulty is not clinically relevant when an experienced anesthetist intubates the trachea in a normal airway condition.
\end{abstract}

Keywords Aerosol box $\cdot$ COVID-19 $\cdot$ Tracheal intubation

\section{Introduction}

On March 11, 2020, the World Health Organization declared that a novel coronavirus disease 2019 (COVID-19) had become a worldwide pandemic. Severe acute respiratory syndrome-corona virus-2 (SARS-CoV-2), which causes COVID-19, is highly contagious [1], and the highest viral load of SARS-CoV-2 appears in the sputum and upper airway secretions [2]. Procedures during airway management including tracheal intubation may generate aerosols that will increase the risk of transmission [1]. Therefore, airway managers should take appropriate precautions [1]. A recent report suggested the usefulness of an aerosol box for protection of the operator during tracheal intubation [3]. However, the effect of the aerosol box on tracheal intubation difficulty is unknown. The aim of this study was to determine the effect of an aerosol box on tracheal intubation difficulty in a normal airway condition.

Ryo Wakabayashi

waka0924@shinshu-u.ac.jp

1 Department of Anesthesiology and Resuscitology, Shinshu University School of Medicine, 3-1-1, Asahi, Matsumoto, Nagano 390-8621, Japan

\section{Methods}

This study was approved by the Committee for Medical Ethics of Shinshu University School of Medicine (No. 4750) and registered in University hospital Medical Information Network Clinical Trials Registry (UMIN000040496). Written informed consent was obtained from all participants before enrollment.

Anesthetists who had performed more than 50 orotracheal intubations using both a direct laryngoscope and a videolaryngoscope and who had normal or corrected-tonormal eyesight were eligible for participation. Eligible anesthetists who refused to participate were excluded. Each participant intubated the trachea of a standard airway manikin (airway management simulator BT-CSIE; BT Inc., Goyang-si, Republic of Korea) 6 times as follows: (1) using a direct laryngoscope and a Macintosh's blade size 4 (HEINE Optotechnik GmbH \& Co. KG, Herrsching, Germany) without an aerosol box; (2) using a direct laryngoscope with an aerosol box; (3) using a McGRATH ${ }^{\mathrm{TM}}$ MAC videolaryngoscope and a McGRATH ${ }^{\mathrm{TM}}$ MAC 4 disposable videolaryngoscope blade (Covidien, Medtronic Inc., Dublin, Ireland) without an aerosol box; (4) using a McGRATH ${ }^{\mathrm{TM}}$ MAC videolaryngoscope with an aerosol box; (5) using an airway scope AWS-S200NK 
videolaryngoscope and an NK PBLADE ITL-SL (Nihon Kohden Corporation, Tokyo, Japan) without an aerosol box; and (6) using an airway scope AWS-S200NK videolaryngoscope with an aerosol box. The aerosol box was made with reference to a previous report (length, $40 \mathrm{~cm}$; height, $50 \mathrm{~cm}$; width, $50 \mathrm{~cm}$; diameter of the circular opening for insertion of arms, $10 \mathrm{~cm}$; position of the circular opening, $25 \mathrm{~cm}$ from base and $5 \mathrm{~cm}$ from the side of the box) [3]. A Shiley ${ }^{\mathrm{TM}}$ tracheal tube (Covidien, Medtronic Inc., Dublin, Ireland) with an internal diameter of $8.0 \mathrm{~mm}$ was used in all intubations. A malleable stylet (Shiley ${ }^{\mathrm{TM}}$ satin slip intubating stylet, Covidien, Medtronic Inc., Dublin, Ireland) was used for tracheal intubation with a direct laryngoscope and a McGRATH ${ }^{\mathrm{TM}}$ MAC videolaryngoscope. An incompressible headrest with a height of $7 \mathrm{~cm}$ was placed beneath the manikin's head and neck to obtain a "sniffing position". The entire bed remained horizontal and the manikin nose-floor distance was set at $105 \mathrm{~cm}$. Each participant was given the opportunity to perform 2 intubations in each setting prior to the study. The order of intubations for each participant was randomly determined by computer-generated randomization using sealed opaque envelopes. The participants did not wear personal protective equipment (PPE) except for standard gloves to conserve PPE supplies. This was because valuable PPE should not be wasted during the COVID-19 outbreak. The airway assistant for all tracheal intubations was a single experienced anesthetist.

The primary outcome was the time to successful tracheal intubation, defined as the time from initial insertion of the laryngoscope in the mouth to the first lung inflation with positive pressure breaths using an anesthesia bag through the tracheal tube with an inflated cuff. Secondary outcomes included first-pass intubation success rate, Cormack-Lehane grade, percentage of glottic opening (POGO) score [4], peak force to maxillary incisors that was measured by force sensors attached to the manikin as default. Failed tracheal intubation was defined as a time for intubating attempt of more than $60 \mathrm{~s}$ or esophageal intubation. The time to successful tracheal intubation and the peak force to maxillary incisors were recorded by a single assistant who was not involved in the study design. The Cormack-Lehane grade and POGO score were evaluated by each intubating anesthetist.

The sample size was determined by using our pilot data $(n=6)$. In our pilot study, the mean (standard deviation) times to successful tracheal intubation were 15 (11), 15 (9), and 14 (8) s when using a direct laryngoscope, a McGRATH $^{\mathrm{TM}}$ MAC videolaryngoscope, and an airway scope AWS-S200NK videolaryngoscope without the aerosol box. Assuming that the aerosol box would prolong the time to successful intubation for $10 \mathrm{~s}$, with type I error set at $5 \%$ and type II error set at $20 \%$, at least 18 participants were required. We scheduled to recruit 20 participants to accommodate a $10 \%$ dropout rate.

Binary and categorial data were analyzed using the chisquared test or Fisher's exact test. Continuous data were checked for normality by the Shapiro-Wilk $W$ test. Normal variables were analyzed using the Student's paired $t$ test or one-way repeated measures one-way analysis of variance with correction for multiple testing by the Bonferroni test. Non-normal variables were analyzed using the Wilcoxon matched pairs signed-rank test or Friedman test with correction for multiple testing by the Dunn test. A $p$ value of less than 0.05 was considered statistically significant. All analyses were performed using GraphPad Prism 7 (GraphPad Software Inc., San Diego, CA, USA).

\section{Results}

Twenty anesthetists were assessed for eligibility and 2 anesthetists were excluded, because they refused to participate. A total of 108 tracheal intubations performed by 18 experienced anesthetists were analyzed. Table 1 shows the intubation outcomes with and without the aerosol box. First-attempt intubation success was achieved in all tracheal intubations. The aerosol box significantly prolonged the time to successful intubation and decreased POGO score when using a direct laryngoscope. When a McGRATH ${ }^{\mathrm{TM}} \mathrm{MAC}$ and an AWS-S200NK were used, the times to successful intubation and POGO scores were comparable with and without the aerosol box. When using any of the laryngoscopes, there were no statistically significant differences in the Cormack-Lehane grade and peak force to maxillary incisors with and without the aerosol box.

Compared with direct laryngoscopy, the times to successful intubation were significantly shorter when a McGRATH $^{\text {TM }}$ MAC and an AWS-S200NK were used in tracheal intubation with the aerosol box $(p<0.001$ and $p=0.003$, respectively). The Cormack-Lehane grade was significantly better when using an AWS-S200NK than when using a direct laryngoscope in tracheal intubation with the aerosol box $(p=0.016)$. The peak forces to maxillary incisors were significantly higher when a direct laryngoscope and a McGRATH ${ }^{\mathrm{TM}}$ MAC were used than when using an AWS-S200NK in tracheal intubation with the aerosol box $(p=0.003$ and $p=0.019$, respectively).

\section{Discussion}

This is the first study in which the effect of an aerosol box on tracheal intubation difficulty was investigated. There have been concerns about possible adverse effects of an aerosol box on tracheal intubation difficulty and patient 
Table 1 Intubation outcomes with and without an aerosol box in a normal airway condition

\begin{tabular}{|c|c|c|c|c|c|c|c|c|c|}
\hline & \multicolumn{2}{|c|}{ Direct laryngoscope } & \multirow[t]{2}{*}{$P$ value } & \multicolumn{2}{|c|}{ McGRATH MAC } & \multirow[t]{2}{*}{$P$ value } & \multicolumn{2}{|l|}{ AWS-S200NK } & \multirow[t]{2}{*}{$P$ value } \\
\hline & $\begin{array}{l}\text { Without AB } \\
n=18\end{array}$ & $\begin{array}{l}\text { With AB } \\
n=18\end{array}$ & & $\begin{array}{l}\text { Without AB } \\
n=18\end{array}$ & $\begin{array}{l}\text { With AB } \\
n=18\end{array}$ & & $\begin{array}{l}\text { Without AB } \\
n=18\end{array}$ & $\begin{array}{l}\text { With AB } \\
n=18\end{array}$ & \\
\hline $\begin{array}{l}\text { Time to suc- } \\
\text { cessful intu- } \\
\text { bation (s) }\end{array}$ & $14(3)$ & $17(4)$ & $<0.001$ & $14(4)$ & $14(4)$ & 0.238 & $13(4)$ & $14(5)$ & 0.084 \\
\hline $\begin{array}{l}\text { Frist-pass } \\
\text { intubation } \\
\text { success }\end{array}$ & $18(100 \%)$ & $18(100 \%)$ & 1.000 & $18(100 \%)$ & $18(100 \%)$ & 1.000 & $18(100 \%)$ & $18(100 \%)$ & 1.000 \\
\hline $\begin{array}{l}\text { Cormack- } \\
\text { Lehane grade }\end{array}$ & & & 0.137 & & & 1.000 & & & 1.000 \\
\hline 1 & $15(83 \%)$ & $11(61 \%)$ & & $16(89 \%)$ & $16(89 \%)$ & & $17(94 \%)$ & $17(94 \%)$ & \\
\hline $2 \mathrm{a}$ & $3(17 \%)$ & $7(39 \%)$ & & $2(11 \%)$ & $2(11 \%)$ & & $1(6 \%)$ & $1(6 \%)$ & \\
\hline $2 b$ & $0(0 \%)$ & $0(0 \%)$ & & $0(0 \%)$ & $0(0 \%)$ & & $0(0 \%)$ & $0(0 \%)$ & \\
\hline 3 & $0(0 \%)$ & $0(0 \%)$ & & $0(0 \%)$ & $0(0 \%)$ & & $0(0 \%)$ & $0(0 \%)$ & \\
\hline 4 & $0(0 \%)$ & $0(0 \%)$ & & $0(0 \%)$ & $0(0 \%)$ & & $0(0 \%)$ & $0(0 \%)$ & \\
\hline POGO score & $100(100-100)$ & $100(70-100)$ & 0.016 & $100(100-100)$ & $100(100-100)$ & 0.500 & $100(100-100)$ & $100(100-100)$ & $>0.999$ \\
\hline $\begin{array}{l}\text { Peak force to } \\
\text { maxillary } \\
\text { incisors }(\mathrm{N})\end{array}$ & & & 0.739 & & & 0.494 & & & 1.000 \\
\hline$<20$ & $9(50 \%)$ & $10(56 \%)$ & & $10(56 \%)$ & $12(67 \%)$ & & $17(94 \%)$ & $18(100 \%)$ & \\
\hline $20-39$ & $9(50 \%)$ & $8(44 \%)$ & & $8(44 \%)$ & $6(33 \%)$ & & $1(6 \%)$ & $0(0 \%)$ & \\
\hline$\geq 40$ & $0(0 \%)$ & $0(0 \%)$ & & $0(0 \%)$ & $0(0 \%)$ & & $0(0 \%)$ & $0(0 \%)$ & \\
\hline
\end{tabular}

Data are expressed as mean (standard deviation), number (proportion), or median (interquartile range)

$A B$ aerosol box, $P O G O$ percentage of glottic opening

safety [5, 6]. In this study, the time to successful intubation was prolonged and POGO score was worsened by the aerosol box when using a direct laryngoscope. However, the statistically significant differences were not clinically relevant. The differences of the time to successful intubation between the types of laryngoscope in tracheal intubation with the aerosol box were also clinically irrelevant. Therefore, our results indicate that the aerosol box has almost no effect on the difficulty in tracheal intubation regardless of the type of laryngoscope when an experienced anesthetist intubates the trachea in a normal airway condition.

There were some limitations in this study. First, full PPE was not used despite the recommendation for wearing full PPE during tracheal intubation in patients with COVID-19 [1]. It has been reported that fogging of goggles and/or glasses may complicate tracheal intubation in up to $80 \%$ of cases [1]. Second, the effect of an aerosol box in a difficult airway setting was not investigated. An aerosol box may have an especially important role as an adjunct to PPE in a difficult airway condition, because prolonged procedures during airway management can generate a larger amount of aerosols. Further studies are required to determine whether an aerosol box affects tracheal intubation difficulty and compromises patient safety in a difficult airway setting.
In conclusion, our results indicate that the effect of an aerosol box on tracheal intubation difficulty is clinically irrelevant when an experienced anesthetist intubates the trachea in a normal airway condition.

Acknowledgements We would like to acknowledge the staff of the Department of Anesthesiology and Resuscitology, Shinshu University School of Medicine, who participated in this study.

\section{Compliance with ethical standards}

Conflict of interest The authors declare that they have no conflict of interest.

\section{References}

1. Cook TM, El-Boghdadly K, McGuire B, McNarry AF, Patel A, Higgs A. Consensus guidelines for managing the airway in patients with COVID-19: Guidelines from the Difficult Airway Society, the Association of Anaesthetists the Intensive Care Society, the Faculty of Intensive Care Medicine and the Royal College of Anaesthetists. Anaesthesia. 2020;75:785-99.

2. Wang W, Xu Y, Gao R, Lu R, Han K, Wu G, Tan W. Detection of SARS-CoV-2 in different types of clinical specimens. JAMA. 2020;323:1843-4. 
3. Canelli R, Connor CW, Gonzalez M, Nozari A, Ortega R. Barrier enclosure during endotracheal intubation. N Engl J Med. 2020;382:1957-8.

4. Levitan RM, Ochroch EA, Kush S, Shofer FS, Hollander JE. Assessment of airway visualization: validation of the percentage of glottic opening (POGO) scale. Acad Emerg Med. 1998;5:919-23.

5. Kearsley R. Intubation boxes for managing the airway in patients with COVID-19. Anaesthesia. 2020;75:962-77.

6. Gould CL, Alexander PDG, Allen CN, McGrath BA, Shelton CL. Protecting staff and patients during airway management in the
COVID-19 pandemic: are intubation boxes safe? Br J Anaesth. 2020. https://doi.org/10.1016/j.bja.2020.05.001.

Publisher's Note Springer Nature remains neutral with regard to jurisdictional claims in published maps and institutional affiliations. 